\title{
Heavy Meson Masses in Chiral Perturbation Theory With Heavy Quark Symmetry
}

\author{
Elizabeth Jenkins* \\ CERN TH Division, CH-1211 Geneva 23, Switzerland
}

\begin{abstract}
The $S U(3)$ and hyperfine mass splittings of mesons containing a single heavy quark are computed to one-loop order in chiral perturbation theory with heavy quark spin symmetry. Electromagnetic contributions of order $\alpha_{e m}$ are included. The observed values of the mass splittings are consistent with the one-loop chiral perturbation theory calculation. The $d-u$ hyperfine splitting $\left(D^{*+}-D^{+}\right)-\left(D^{* 0}-D^{0}\right)$ is equal to $\left(m_{d}-m_{u}\right) / m_{s}$ times the $s-d$ hyperfine splitting $\left(D_{s}^{*+}-D_{s}^{+}\right)-\left(D^{*+}-D^{+}\right)$upto electromagnetic contributions. The small observed value of the $s-d$ hyperfine splitting implies that the $d-u$ hyperfine splitting is completely dominated by electromagnetic mass contributions.
\end{abstract}

CERN-TH.6765/92

hep-ph/9212295

December 1992

* On leave from the University of California at San Diego. 


\section{Introduction}

The spectrum of masses for the lowest-lying pseudoscalar and vector mesons which contain a single heavy quark has been of recent theoretical interest. Two approximate symmetries of QCD, chiral symmetry for the light $u, d$ and $s$ quarks and heavy quark spin-flavor symmetry [1] for the heavy $c$ and $b$ quarks, can be exploited to calculate the heavy meson masses. These symmetries have led to a number of theoretical predictions for the heavy meson masses. The hyperfine mass splittings $D^{*}-D$ and $B^{*}-B$ are related using heavy quark spin-flavor symmetry. Corrections to the naive $1 / m_{Q}$ scaling of these splittings can be calculated in HQET [2]. The $S U(3)$ flavor splitting $D_{s}-D$ can be calculated using chiral Lagrangian techniques. A leading nonanalytic chiral correction to this mass splitting is calculated in ref. [3]. Rosner and Wise [4] recently analyzed the heavy meson mass spectrum to first order in light quark masses and to order $1 / m_{Q}$. The leading effects from electromagnetism proportional to one power of $\alpha_{e m}$ or $\alpha_{e m} / m_{Q}$ also were considered. Rosner and Wise obtain the mass relation $\left\{\left(B_{s}^{*}-B_{s}\right)-\left(B^{* 0}-B^{0}\right)\right\}=$ $\left(m_{c} / m_{b}\right)\left\{\left(D_{s}^{*}-D_{s}\right)-\left(D^{*+}-D^{+}\right)\right\}$. The electromagnetic splittings of the mesons have been estimated using dispersion relation techniques [5]. Estimates of the hyperfine mass splittings for the $D$ and $B$ mesons are discussed in ref. [6].

In this work, the masses of the $D, D^{*}, B$ and $B^{*}$ mesons are calculated to one-loop order in chiral perturbation theory using a chiral Lagrangian which incorporates heavy quark spin-flavor symmetry and its breaking. The calculation naturally breaks up into the calculation of $S U(3)$ mass splittings which preserve heavy quark spin symmetry and the calculation of hyperfine mass splittings which explicitly violate heavy quark spin symmetry. The one-loop chiral perturbation theory calculation results in contributions to the meson masses which are nonanalytic in the light quark masses. The leading contribution to the $s-d$ hyperfine mass difference $\left(D_{s}^{*+}-D_{s}^{+}\right)-\left(D^{*+}-D^{+}\right)$is nonanalytic in the light quark masses. The small measured value of this hyperfine mass difference implies nearly exact cancellation between the nonanalytic contribution and a one-loop counterterm. The contribution to the $d-u$ hyperfine mass splitting $\left(D^{*+}-D^{+}\right)-\left(D^{* 0}-D^{0}\right)$ due to

light quark mass differences is simply equal to the $s-d$ mass splitting times the ratio $\left(m_{s}-m_{d}\right) /\left(m_{d}-m_{u}\right)$. Experimentally, the hyperfine mass differences do not satisfy such a relation; both hyperfine mass splittings are of order one MeV. Thus, the $d-u$ hyperfine mass difference must be dominated by electromagnetic effects. (There is no electromagnetic contribution to the $s-d$ hyperfine mass difference since the $s$ and $d$ quarks have equal 
charges.) Finally, nonanalytic corrections do not violate the Rosner-Wise mass relation. The relation is violated at order $\left(1 / m_{Q}\right)^{2}$ in the heavy quark mass expansion.

The organization of this paper is as follows. The chiral Lagrangian for heavy mesons is constructed in Sect. 2. The leading order Lagrangian for heavy meson-pion interactions which satisfies heavy quark spin-flavor symmetry is reviewed. Higher order terms in the chiral Lagrangian which are relevant for the calculation of the heavy meson mass splittings are discussed next. These terms break chiral symmetry and heavy quark symmetry. The terms are classified by the number of insertions of the light quark mass matrix they contain and by whether or not they violate the heavy quark spin symmetry. Finally, chiral Lagrangian terms describing the effects of electromagnetism on the heavy meson masses are detailed. Only the leading operators which are suppressed by a single power of $\alpha_{e m}$ are included. Electromagnetic effects break chiral symmetry and heavy quark spin-flavor symmetry since the charges and magnetic moments of the light and heavy quarks violate these symmetries. Sect. 3 contains the calculation of the $S U(3)$ and hyperfine mass splittings of the heavy mesons to one-loop order in chiral perturbation theory neglecting the masses of the $u$ and $d$ quarks and electromagnetic effects. Sect. 4 generalizes the calculation to include nonvanishing $u$ and $d$ quark masses, as well as electromagnetic corrections. In Sect. 5, the theoretical formulæ for the mass splittings are compared with experiment. Sect. 6 contains final remarks.

\section{Chiral Lagrangian for Heavy Mesons}

The lowest lying mesons which contain a single heavy quark $Q$ are pseudoscalar and vector mesons with spin of the light degrees of freedom $s_{\ell}=\frac{1}{2}$. Degenerate spin zero and spin one mesons result when the spin of the light degrees of freedom is combined with the spin of the heavy quark $s_{Q}=\frac{1}{2}$. Since the pseudoscalar and vector mesons are degenerate in the heavy quark mass limit and the spin of the heavy quark is conserved by low energy strong interactions, it is convenient to introduce a single field [7] for the heavy meson multiplet 1 ,

$$
H_{a}^{(Q)}=\frac{(1+\psi)}{2}\left[P_{a \mu}^{*(Q)} \gamma^{\mu}-P_{a}^{(Q)} \gamma_{5}\right] \frac{(1-\psi)}{2}
$$

1 The usual definition of the $4 \times 4$ Dirac matrix $H_{a}^{(Q)}$ does not include the projector $(1-\not) / 2$. This projector, however, results from commuting the heavy quark velocity projector $(1+\psi) / 2$ through the matrix in square brackets since $v \cdot P^{*}=0$. 
where $P_{a}^{(Q)}$ and $P_{a}^{*(Q)}$ are the pseudoscalar and vector mesons fields, respectively, with flavor quantum numbers $Q \bar{q}_{a}$ where $Q=c, b$ and $a=1,2,3$ (or $u, d, s$ ). For $Q=c$, these fields are the $\left(D_{1}, D_{2}, D_{3}\right)=\left(D^{0}, D^{+}, D_{s}\right)$ and $\left(D_{1}^{*}, D_{2}^{*}, D_{3}^{*}\right)=\left(D^{* 0}, D^{*+}, D_{s}^{*}\right)$ mesons, whereas for $Q=b$, they are the $\left(B_{1}, B_{2}, B_{3}\right)=\left(B^{-}, \bar{B}^{0}, \bar{B}_{s}\right)$ and $\left(B_{1}^{*}, B_{2}^{*}, B_{3}^{*}\right)=\left(B^{*-}, \bar{B}^{* 0}, \bar{B}_{s}^{*}\right)$ mesons. The heavy meson field (2.1) also carries a velocity subscript which has been suppressed. The velocity-dependent field [8] is defined by the field redefinition

$$
H_{v}^{(Q)}=\sqrt{m_{H}} e^{i m_{H} \psi v_{\mu} x^{\mu}} H^{(Q)}(x),
$$

which removes the large mass $m_{H} \operatorname{Tr} \bar{H} H$ from the HQET Lagrangian. The velocitydependent field satisfies $\psi H_{v}=H_{v}$ and $H_{v} \psi=-H_{v}$. The conjugate heavy meson field is defined by

$$
\bar{H}^{a(Q)}=\gamma^{0} H_{a}^{(Q) \dagger} \gamma^{0}=\frac{(1-\psi)}{2}\left[P_{\mu}^{* a(Q) \dagger} \gamma^{\mu}+P^{a(Q) \dagger} \gamma_{5}\right] \frac{(1+\psi)}{2}
$$

The low energy interactions of mesons containing a single heavy quark with pions are described by a chiral Lagrangian which respects heavy quark spin-flavor symmetry [9] [10] [11]. The heavy meson field $H_{v}$ transforms as a doublet under heavy quark spin symmetry and as a $\overline{3}$ under flavor $S U(3)_{V}$,

$$
H_{a}^{(Q)} \rightarrow S_{Q}\left(H^{(Q)} U^{\dagger}\right)_{a}
$$

In addition, the heavy meson field transforms as a doublet under heavy quark flavor symmetry. The field redefinition which defines the velocity-dependent field $H_{v}$ removes the singlet mass term, the mass which is invariant under chiral symmetry and heavy quark spin-flavor symmetry, from the chiral Lagrangian. The chiral Lagrangian which is formulated using this velocity-dependent field has a valid derivative expansion and loop expansion because the chiral Lagrangian has a systematic expansion in $1 / m_{H}\left(\right.$ or $\left.1 / m_{Q}\right)$ [12] [13]. Derivatives acting on the heavy meson field produce factors of $k$, the residual off-shell momentum of the heavy meson field, or equivalently, the typical momentum of the pions interacting with the heavy mesons. Higher dimensional terms of the chiral Lagrangian are suppressed by powers of either $1 / m_{H}$ or $1 / \Lambda_{\chi}$, where $\Lambda_{\chi} \sim 1 \mathrm{GeV}$ is the chiral symmetry breaking scale [14. Note that the value of $m_{H}$ used to define velocity-dependent heavy meson fields will be quite different for the $D^{(*)}$ and $B^{(*)}$ mesons. The mass $m_{H}$ can be expanded in a power series in $m_{Q}$. To leading order in this expansion, $m_{H}=m_{Q}$. 
The leading order chiral Lagrangian for heavy mesons interacting with pions is given by

$$
\mathcal{L}_{v}^{0}=-i \operatorname{Tr} \bar{H}_{v}(v \cdot \partial) H_{v}+i \operatorname{Tr} \bar{H}_{v} H_{v}(v \cdot V)+2 g \operatorname{Tr} \bar{H}_{v} H_{v}\left(S_{\ell v} \cdot A\right),
$$

where the heavy and light quark flavor quantum numbers of the heavy meson field have been suppressed. In eq. (2.5), all traces are taken over Dirac spinor indices, light quark $S U(3)_{V}$ flavor indices $a=u, d, s$, and heavy quark flavor indices $Q=c, b$. The pion octet

$$
\pi=\frac{1}{\sqrt{2}}\left(\begin{array}{ccc}
\frac{1}{\sqrt{2}} \pi^{0}+\frac{1}{\sqrt{6}} \eta & \pi^{+} & K^{+} \\
\pi^{-} & -\frac{1}{\sqrt{2}} \pi^{0}+\frac{1}{\sqrt{6}} \eta & K^{0} \\
K^{-} & \bar{K}^{0} & -\frac{2}{\sqrt{6}} \eta
\end{array}\right),
$$

appears in Lagrangian $\mathcal{L}_{v}^{0}$ through the vector and axial vector linear combinations

$$
V^{\mu}=\frac{1}{2}\left(\xi \partial^{\mu} \xi^{\dagger}+\xi^{\dagger} \partial^{\mu} \xi\right), \quad A^{\mu}=\frac{i}{2}\left(\xi \partial^{\mu} \xi^{\dagger}-\xi^{\dagger} \partial^{\mu} \xi\right),
$$

where

$$
\xi=e^{i \pi / f}, \quad \Sigma=\xi^{2}=e^{2 i \pi / f},
$$

and $f \approx 93 \mathrm{MeV}$ is the pion decay constant. Under $S U(3)_{L} \times S U(3)_{R}$ chiral symmetry,

$$
\Sigma \rightarrow L \Sigma R^{\dagger}, \quad \xi \rightarrow L \xi U^{\dagger}=U \xi R^{\dagger},
$$

where $U$ is defined by the transformation of $\xi$. The Dirac structure of the heavy meson chiral Lagrangian has been replaced by the velocity vector $v^{\mu}$ and the velocity-dependent spin operator $S_{\ell v}^{\mu}$ for the light degrees of freedom [12]. The spin operator for the light degrees of freedom is defined by $\operatorname{Tr} \bar{H}_{v} H_{v} \gamma^{\mu} \gamma_{5} \equiv 2 \operatorname{Tr} \bar{H}_{v} H_{v} S_{\ell v}^{\mu}$, and satisfies the identity $\left(v \cdot S_{\ell v}\right)=0$. In the rest frame $v^{\mu}=(1, \overrightarrow{0})$ of the heavy meson, $S_{\ell}^{\mu}=(0, \vec{\sigma} / 2)$. The axial vector combination of pions $A^{\mu}=\partial^{\mu} \pi / f+\ldots$ couples to the spin of the light degrees of freedom in the heavy mesons. The spin of the heavy quark is unaffected by the low energy pion interactions of the heavy mesons, however, since an axial vector coupling to the spin of the heavy quark $\operatorname{Tr} \bar{H}_{v} A \gamma_{5} H_{v} \equiv 2 \operatorname{Tr} \bar{H}_{v}\left(S_{Q v} \cdot A\right) H_{v}$ is forbidden by heavy quark spin symmetry. Thus, the chiral Lagrangian $\mathcal{L}_{v}^{0}$ depends on only a single axial vector coupling constant $g$. The coupling $g$ is constrained by the radiative and pion decay widths of the vector mesons, $g^{2} \lesssim 0.5$ [15] [16] [17]. The propagator of the $H_{v}$ field is easily determined from the leading order chiral Lagrangian to be $-i /(k \cdot v)$. Note that $H_{v}$ is normalized to unity which implies that the fields $P$ and $P^{*}$ are normalized to 2, 
since $\operatorname{Tr} \bar{H}_{v} H_{v}=2\left(P_{a \mu}^{* \dagger} P_{a}^{* \mu}-P_{a}^{\dagger} P_{a}\right)$. Loop calculations of corrections which respect heavy quark spin symmetry can be calculated using the field $H_{v}$ [18]. Heavy quark spin symmetry violating corrections are best calculated using the fields $P$ and $P^{*}$.

Higher dimensional operators of the chiral Lagrangian which break heavy quark spinflavor symmetry and chiral symmetry involve factors of $1 / m_{Q}$ or insertions of the light quark mass matrix $M=\operatorname{diag}\left(m_{u}, m_{d}, m_{s}\right)$. For the calculation of the heavy meson masses, it is useful to classify the symmetry-violating operators by the number of insertions of the quark mass matrix and whether or not they violate the heavy quark spin symmetry. Operators which respect heavy quark spin symmetry have coefficients which start at $\mathcal{O}(1)$ in the $1 / m_{Q}$ expansion, whereas operators which violate heavy quark spin symmetry have coefficients which start at $\mathcal{O}\left(1 / m_{Q}\right)$. Counterterms to the one-loop calculation of the heavy meson masses are proportional to two powers of the light quark masses. Thus terms with up to two insertions of $M$ are needed for the one-loop calculation.

The singlet mass term $m_{H}$ is a function of $1 / m_{Q}$, but is irrelevant for the calculation of meson mass splittings. There is only one other term with no insertions of the light quark mass matrix,

$$
\mathcal{L}_{v}^{\phi}=-\frac{\Delta}{8} \operatorname{Tr} \bar{H}_{v} \sigma^{\mu \nu} H_{v} \sigma_{\mu \nu}=-\Delta \operatorname{Tr} \bar{H}_{v} S_{Q}^{\alpha}{ }_{v} H_{v} S_{\ell v \alpha} .
$$

This term violates heavy quark spin-flavor symmetry and is responsible for the hyperfine $\left(P^{*}-P\right)$ mass splitting at leading order. The parameter $\Delta$ is a function of $1 / m_{Q}$ which starts at linear order in $1 / m_{Q}$, because violations of heavy quark spin symmetry are suppressed by at least one power of $1 / m_{Q}$. The value of $-S_{Q} \cdot S_{\ell}=\vec{S}_{Q} \cdot \vec{S}_{\ell}=\frac{1}{2}\left(\vec{S}_{T}^{2}-\vec{S}_{Q}^{2}-\vec{S}_{\ell}^{2}\right)$ equals $-\frac{3}{4}$ for pseudoscalar mesons $P$ and $\frac{1}{4}$ for vector mesons $P^{*}$. Thus, this operator results in a hyperfine splitting $\left(P^{*}-P\right)=\Delta$ at tree level. Note that the Dirac tensor structure $\operatorname{Tr} \bar{H}_{v} \sigma^{\mu \nu} H_{v} \sigma_{\mu \nu}$ is the only possible spin symmetry-violating Lorentz invariant since the operators $\operatorname{Tr} \bar{H}_{v} \sigma^{\mu \nu} \sigma_{\mu \nu} H_{v}$ and $\operatorname{Tr} \bar{H}_{v} H_{v} \sigma^{\mu \nu} \sigma_{\mu \nu}$ are proportional to $\operatorname{Tr} \bar{H}_{v} H_{v}$ because $\vec{S}_{Q}^{2}$ and $\vec{S}_{\ell}^{2}$ are constants for the heavy meson field.

The terms in the chiral Lagrangian which are proportional to a light quark mass and which respect the heavy quark spin symmetry are given by

$$
\mathcal{L}_{v}^{M}=a \operatorname{Tr} \bar{H}_{v} H_{v} M_{\xi}+\sigma \operatorname{Tr} M_{\xi} \operatorname{Tr} \bar{H}_{v} H_{v}
$$

where $a$ and $\sigma$ are functions of $1 / m_{Q}$ which start at $\mathcal{O}(1)$. The term proportional to $a$

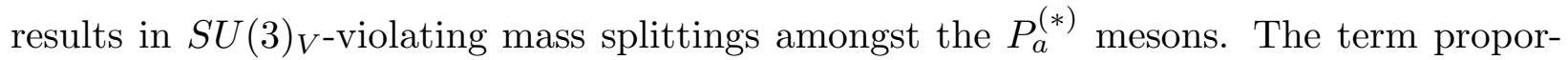
tional to $\sigma$ leads to a singlet contribution to the masses which depends linearly on the light quark masses, and is the heavy meson analog of the pion-nucleon sigma term. 
Chiral Lagrangian terms with one insertion of the light quark mass matrix which violate the heavy quark spin symmetry are

$$
\mathcal{L}_{v}^{M \$}=-\frac{1}{8} \Delta^{(a)} \operatorname{Tr} \bar{H}_{v} \sigma^{\mu \nu} H_{v} \sigma_{\mu \nu} M_{\xi}-\frac{1}{8} \Delta^{(\sigma)} \operatorname{Tr} M_{\xi} \operatorname{Tr} \bar{H}_{v} \sigma^{\mu \nu} H_{v} \sigma_{\mu \nu}
$$

These two terms produce hyperfine splittings proportional to a light quark mass. The term proportional to $\Delta^{(a)}$ leads to light quark flavor-dependent hyperfine splittings, whereas the term proportional to $\Delta^{(\sigma)}$ yields a flavor singlet contribution to the hyperfine splittings which is linear in the light quark masses.

Chiral Lagrangian terms with two insertions of the light quark mass matrix which preserve and violate the heavy quark spin symmetry are

$$
\mathcal{L}_{v}^{M M}=b \operatorname{Tr} \bar{H}_{v} H_{v} M_{\xi} M_{\xi}+c \operatorname{Tr} M_{\xi} \operatorname{Tr} \bar{H}_{v} H_{v} M_{\xi}+d \operatorname{Tr} M_{\xi} M_{\xi} \operatorname{Tr} \bar{H}_{v} H_{v}
$$

and

$$
\begin{aligned}
\mathcal{L}_{v}^{M M \$}= & -\frac{1}{8} \Delta^{(b)} \operatorname{Tr} \bar{H}_{v} \sigma^{\mu \nu} H_{v} \sigma_{\mu \nu} M_{\xi} M_{\xi} \\
& -\frac{1}{8} \Delta^{(c)} \operatorname{Tr} M_{\xi} \operatorname{Tr} \bar{H}_{v} \sigma^{\mu \nu} H_{v} \sigma_{\mu \nu} M_{\xi} \\
& -\frac{1}{8} \Delta^{(d)} \operatorname{Tr} M_{\xi} M_{\xi} \operatorname{Tr} \bar{H}_{v} \sigma^{\mu \nu} H_{v} \sigma_{\mu \nu},
\end{aligned}
$$

respectively. The coefficients $b, c$ and $d$ are functions of $1 / m_{Q}$ which begin at $\mathcal{O}(1)$, whereas the coefficients $\Delta^{(b)}, \Delta^{(c)}$, and $\Delta^{(d)}$ are functions of $1 / m_{Q}$ which begin at $\mathcal{O}\left(1 / m_{Q}\right)$. 2

Electromagnetic mass splittings are incorporated into the chiral Lagrangian framework by including terms which depend quadratically on the charges of the heavy and light quarks, and are suppressed by at least one power of $\alpha_{e m}$. The heavy quark charges $q_{c}=\frac{2}{3}, q_{b}=-\frac{1}{3}$ respect chiral $S U(3)_{V}$ symmetry but break heavy quark flavor symmetry. The light quark charges appear in the chiral Lagrangian through the light quark charge matrix $\mathcal{Q}=\operatorname{diag}\left(\frac{2}{3},-\frac{1}{3},-\frac{1}{3}\right)$ which transforms nontrivially under $S U(3)_{V}$. The light quark charge matrix appears in operators of the chiral Lagrangian in the linear combination $\mathcal{Q}_{\xi}=\frac{1}{2}\left(\xi \mathcal{Q} \xi^{\dagger}+\xi^{\dagger} \mathcal{Q} \xi\right)$, which transforms simply under chiral symmetry, $\mathcal{Q}_{\xi} \rightarrow U \mathcal{Q}_{\xi} U^{\dagger}$. In the following, terms of order $\alpha_{e m}$ with insertions of the quark mass matrix are neglected. The number of chiral invariants constructed with $\mathcal{Q}_{\xi}$ is less than the number for $M_{\xi}$, since $\operatorname{Tr} \mathcal{Q}_{\xi}=\frac{1}{2} \operatorname{Tr}\left(\xi \mathcal{Q} \xi^{\dagger}+\xi^{\dagger} \mathcal{Q} \xi\right)=\operatorname{Tr} \mathcal{Q}=0$.

2 The coefficients which violate heavy quark spin symmetry (i.e. $\Delta, \Delta^{(\sigma)}, \Delta^{(a)}, \Delta^{(b)}, \Delta^{(c)}$, and $\left.\Delta^{(d)}\right)$ also have calculable logarithmic dependence on the heavy quark mass from perturbative QCD [2]. 
Heavy quark flavor symmetry-violating but heavy quark spin symmetry-conserving contributions to the $S U(3)$-invariant mass of the heavy meson multiplet are generated by the operator $\alpha_{e m} q_{Q}^{2} \operatorname{Tr} \bar{H}_{v} H_{v}$ which contains two factors of the heavy quark charge. This term does not contribute to the meson mass splittings, however, and need not be considered here. In addition, there is a term with two factors of the heavy quark charge which violates the heavy quark spin and flavor symmetries,

$$
\mathcal{L}_{v}^{q_{Q} q_{Q} \$}=-\frac{1}{8} \Delta_{e m} \alpha_{e m} \operatorname{Tr} \bar{H}_{v} q_{Q}^{2} \sigma^{\mu \nu} H_{v} \sigma_{\mu \nu}
$$

and which contributes differently to the $S U(3)_{V}$ invariant hyperfine splittings for the $D$ and $B$ mesons. Electromagnetic contributions from terms containing one factor of the light quark charge matrix and one factor of the heavy quark charge are given by

$$
\mathcal{L}_{v}^{q_{Q} Q}=a_{e m} \alpha_{e m} \operatorname{Tr} \bar{H}_{v} q_{Q} H_{v} \mathcal{Q}_{\xi},
$$

and

$$
\mathcal{L}_{v}^{q_{Q} \mathcal{Q} \$}=-\frac{1}{8} \Delta_{e m}^{(a)} \alpha_{e m} \operatorname{Tr} \bar{H}_{v} q_{Q} \sigma^{\mu \nu} H_{v} \mathcal{Q}_{\xi} \sigma_{\mu \nu},
$$

which preserve and violate the heavy quark spin symmetry, respectively. The terms in $\mathcal{L}_{v}^{q_{Q} Q}$ and $\mathcal{L}_{v}^{q_{Q} Q \$}$ violate heavy quark flavor symmetry and isospin. Terms in the chiral Lagrangian with two insertions of the light quark mass matrix are given by

$$
\mathcal{L}_{v}^{\mathcal{Q Q}}=b_{e m} \alpha_{e m} \operatorname{Tr} \bar{H}_{v} H_{v} \mathcal{Q}_{\xi} \mathcal{Q}_{\xi}+d_{e m} \alpha_{e m} \operatorname{Tr} \mathcal{Q}_{\xi} \mathcal{Q}_{\xi} \operatorname{Tr} \bar{H}_{v} H_{v}
$$

which respects heavy quark symmetry, and by

$$
\begin{aligned}
\mathcal{L}_{v}^{Q Q \Phi}= & -\frac{1}{8} \Delta_{e m}^{(b)} \alpha_{e m} \operatorname{Tr} \bar{H}_{v} \sigma^{\mu \nu} H_{v} \sigma_{\mu \nu} \mathcal{Q}_{\xi} \mathcal{Q}_{\xi} \\
& -\frac{1}{8} \Delta_{e m}^{(d)} \alpha_{e m} \operatorname{Tr} \mathcal{Q}_{\xi} \mathcal{Q}_{\xi} \operatorname{Tr} \bar{H}_{v} \sigma^{\mu \nu} H_{v} \sigma_{\mu \nu},
\end{aligned}
$$

which violates heavy quark spin symmetry. The first terms in $\mathcal{L}_{v}^{Q Q}$ and $\mathcal{L}_{v}^{Q Q \phi}$ violate isospin.

The electromagnetic mass terms eq. (2.16) and eq. (2.18) are expected to be of order 2-3 MeV, which is the size of typical electromagnetic contributions to hadron masses. The electromagnetic hyperfine mass terms eqs. (2.15), (2.17) and (2.19) are due to the electromagnetic hyperfine interaction between the light and heavy quarks, and are expected to be of order $\Lambda / m_{Q}$ times the spin-independent electromagnetic splitting. Here $\Lambda$ denotes a typical hadronic scale, which in a quark model is of order a constituent quark mass of 350-550 MeV. Thus, eq. (2.15), (2.17) and (2.19) each contribute $0.5-1 \mathrm{MeV}$ to the $D$ meson masses and $0.2-0.3 \mathrm{MeV}$ to the $B$ meson masses. Electromagnetic contributions are relevant only for the isospin and isospin hyperfine splittings of the heavy mesons. 


\section{Calculation of Heavy Meson Masses Neglecting $m_{u}$ and $m_{d}$}

In this section, the heavy meson masses are calculated to one-loop order in chiral perturbation theory with $m_{u}=m_{d}=0$ and $\alpha_{e m}=0$. In this limit, isospin is an exact symmetry, and electromagnetic contributions to the masses are ignored. Since the strange quark is much heavier than the $u$ and $d$ quarks and $m_{s}-m_{u} \approx m_{s}-m_{d} \approx m_{s}, S U(3)_{V}$ splittings can be determined by neglecting the $u$ and $d$ quark masses relative to the strange quark mass. Isospin splittings will be considered in the next section where the calculation is generalized to include nonvanishing $u$ and $d$ quark masses. 目

The one-loop calculation of the heavy meson masses naturally separates into oneloop calculations for two different linear combinations of the masses. The first linear combination $\frac{1}{4}\left(P_{a}+3 P_{a}^{*}\right)$ respects heavy quark spin symmetry and yields the purely $S U(3)_{V}$-symmetric and $S U(3)_{V}$-violating contributions to the meson masses. The second linear combination $\left(P_{a}^{*}-P_{a}\right)$ violates heavy quark spin symmetry and yields the $S U(3)_{V}$-symmetric and $S U(3)_{V}$-violating contributions to the hyperfine mass splittings of the heavy mesons.

To one-loop, the chiral expansions for these two linear combinations of the heavy meson masses can be written in the form ${ }^{\theta}$

$$
\frac{1}{4}\left(P_{a}+3 P_{a}^{*}\right)=m_{H}+\alpha_{a}-\beta_{a} \frac{M_{K}^{3}}{16 \pi f^{2}}+\left(\gamma_{a}-\lambda_{a} \alpha_{a}\right) \frac{M_{K}^{2}}{16 \pi^{2} f^{2}} \ln \left(M_{K}^{2} / \mu^{2}\right)+c_{a},
$$

and

$$
\left(P_{a}^{*}-P_{a}\right)=\Delta+\left(\gamma_{a}-\lambda_{a} \Delta\right) \frac{M_{K}^{2}}{16 \pi^{2} f^{2}} \ln \left(M_{K}^{2} / \mu^{2}\right)+\delta c_{a},
$$

where $\alpha_{a}$, the tree-level mass splittings calculated from $\mathcal{L}_{v}^{M}$, are shown in fig. 1; $\lambda_{a}$ describes wavefunction renormalization,

$$
Z_{a}=1+\lambda_{a}\left(M_{K}^{2} / 16 \pi^{2} f^{2}\right) \ln \left(M_{K}^{2} / \mu^{2}\right)
$$

$\beta_{a}$ parametrizes the nonanalytic $m_{s}^{3 / 2}$ contribution; $\gamma_{a}$ describes the nonanalytic chiral logarithmic corrections from the one-loop graphs shown in fig. 2; $c_{a}$ are contact terms for

3 Electromagnetic effects do not contribute to $S U(3)_{V}$ breaking mass splittings amongst mesons carrying $d$ and $s$ light flavor quantum numbers since the charges of the $d$ and $s$ quarks are the same. Thus, if one restricts the formulæ given in this section to these mesons, all $S U(3)_{V^{-}}$ violating contributions are taken into account. Neglected electromagnetic contributions still result in small heavy quark flavor symmetry violation, however.

4 Analogous formulæ are obtained for the baryon octet and decuplet masses [19] [20] [13]. 
the spin-independent contributions to the masses arising from $\mathcal{L}_{v}^{M M}$; and $\delta c_{a}$ are contact terms for the hyperfine splittings arising from $\mathcal{L}_{v}^{M \$}$. Only the kaon mass $M_{K}$ appears in eqs. (3.1) and (3.2) since the pion mass vanishes for $m_{u}=m_{d}=0$ and the $\eta$ mass can be eliminated using the Gell-Mann-Okubo formula $M_{\eta}^{2}=\frac{4}{3} M_{K}^{2}$. The scale $\mu$ is an arbitrary renormalization scale, and is chosen to be $\mu \sim 1 \mathrm{GeV}$. The one-loop expressions eqs. (3.1) and (3.2) including the contact terms are independent of the scale $\mu$, because the $\mu$ dependence of the chiral logarithms is canceled by the $\mu$ dependence of the counterterms. Changing the scale $\mu$ appearing in the chiral logarithms redefines these local contact terms. The chiral expansion for the linear combination $\frac{1}{4}\left(P_{a}+3 P_{a}^{*}\right)$ to one-loop order including contact counterterms includes all contributions to order $m_{s}^{2}$ since the treelevel mass splittings are linear in $m_{s}$. In contrast, the one-loop calculation of the hyperfine mass differences $\left(P_{a}^{*}-P_{a}\right)$ only includes effects to order $m_{s}$ since the leading contribution $\Delta$ to these mass differences is independent of $m_{s}$. Calculation of the hyperfine mass differences to order $m_{s}^{2}$ involves a two-loop renormalization of the tree-level contribution $\Delta$, a one-loop renormalization of the contributions $\Delta^{(\sigma)} m_{s}$ and $\Delta^{(a)} m_{s}$, and contact terms arising from the spin-dependent operators contained in $\mathcal{L}_{v}^{M M \$}$.

The coefficients appearing in eqs. (3.1) and (3.2) are calculated in terms of the parameters given in Sect. 2. The tree-level coefficients are

$$
\alpha_{a}=2 \sigma m_{s}+2 a m_{s} \delta_{3 a}
$$

The coefficients of the $m_{s}^{3 / 2}$ contribution 5 to the masses,

$$
\begin{aligned}
& \beta_{1,2}=g^{2}\left(1+\frac{8}{3 \sqrt{3}} \frac{1}{6}\right) \\
& \beta_{3}=g^{2}\left(2+\frac{8}{3 \sqrt{3}} \frac{2}{3}\right),
\end{aligned}
$$

are obtained from the graph fig. 2(a). This graph also is responsible for the wavefunction renormalization coefficients

$$
\begin{aligned}
& \lambda_{1,2}=\frac{3}{2} g^{2}\left(\frac{11}{9}\right) \\
& \lambda_{3}=\frac{3}{2} g^{2}\left(\frac{26}{9}\right) .
\end{aligned}
$$

5 The $m_{s}^{3 / 2}$ contribution given here differs from the correction given in ref. [3] by an overall factor of $\frac{4}{3}$. The coefficients $\beta_{a}$ are simply related to wavefunction renormalization coefficients $\lambda_{a}$ in the same manner as for baryons [20] [13] since the spin of the light degrees of freedom, which couples to pions, is $\frac{1}{2}$ for both the baryons and the heavy mesons. In the case of the baryons which contain no heavy quarks, the spin of the light degrees of freedom is the total spin of the baryon. 
The coefficient of the chiral logarithmic correction in eq. (3.1) is given by

$$
\left(\gamma_{a}-\lambda_{a} \alpha_{a}\right)= \begin{cases}-2 a m_{s}\left(\frac{1}{2}-\frac{3}{2} g^{2}\right)-2 \sigma m_{s}\left(\frac{26}{9}\right) & a=1,2, \\ -2 a m_{s}\left(\frac{17}{9}+3 g^{2}\right)-2 \sigma m_{s}\left(\frac{26}{9}\right) & a=3\end{cases}
$$

while the counterterms obtained from $\mathcal{L}_{v}^{M M}$ and $\mathcal{L}_{v}^{M M \$}$ are

$$
c_{a}=4 d m_{s}^{2}+\left(4 c m_{s}^{2}+4 b m_{s}^{2}\right) \delta_{3 a} .
$$

The one-loop chiral logarithmic renormalization of $\Delta$ is determined by

$$
\left(\gamma_{a}-\lambda_{a} \Delta\right)=-\Delta\left(\frac{2}{3} \lambda_{a}\right)
$$

whereas the counterterm 6 for this chiral logarithm is

$$
\delta c_{a}=2 \Delta^{(\sigma)} m_{s}+2 \Delta^{(a)} m_{s} \delta_{3 a}
$$

In the isospin limit, there are four heavy meson masses $P, P^{*}, P_{s}$, and $P_{s}^{*}$. These masses are determined by four linear combinations: an $S U(3)_{V}$ symmetric, heavy quark spin-independent contribution $\frac{1}{4}\left(P+3 P^{*}\right)$; an $S U(3)_{V}$-breaking, spin-independent contribution $\left\{\frac{1}{4}\left(P_{s}+3 P_{s}^{*}\right)-\frac{1}{4}\left(P+3 P^{*}\right)\right\}$; an $S U(3)_{V}$ symmetric, heavy quark spindependent contribution $\left(P^{*}-P\right)$; and an $S U(3)_{V}$-violating, spin-dependent contribution $\left\{\left(P_{s}^{*}-P_{s}\right)-\left(P^{*}-P\right)\right\}$. These four linear combinations are given by

$$
\begin{gathered}
\frac{1}{4}\left(P+3 P^{*}\right)=m_{H}+2 \sigma m_{s}-g^{2}\left(1+\frac{8}{3 \sqrt{3}} \frac{1}{6}\right) \frac{M_{K}^{3}}{16 \pi f^{2}} \\
-2 \sigma m_{s}(2) \frac{M_{K}^{2}}{16 \pi^{2} f^{2}} \ln \left(M_{K}^{2} / \mu^{2}\right) \\
-2 \sigma m_{s}\left(\frac{2}{3}\right) \frac{M_{\eta}^{2}}{16 \pi^{2} f^{2}} \ln \left(M_{\eta}^{2} / \mu^{2}\right) \\
-2 a m_{s}\left(\frac{1}{2}-\frac{3}{2} g^{2}\right) \frac{M_{K}^{2}}{16 \pi^{2} f^{2}} \ln \left(M_{K}^{2} / \mu^{2}\right)+4 d m_{s}^{2} \\
\left\{\frac{1}{4}\left(P_{s}+3 P_{s}^{*}\right)-\frac{1}{4}\left(P+3 P^{*}\right)\right\}=2 a m_{s}-g^{2}\left(1+\frac{8}{3 \sqrt{3}} \frac{1}{2}\right) \frac{M_{K}^{3}}{16 \pi f^{2}} \\
-2 a m_{s}\left(\frac{1}{2}+\frac{9}{2} g^{2}\right) \frac{M_{K}^{2}}{16 \pi^{2} f^{2}} \ln \left(M_{K}^{2} / \mu^{2}\right) \\
-2 a m_{s}\left(\frac{2}{3}\right) \frac{M_{\eta}^{2}}{16 \pi^{2} f^{2}} \ln \left(M_{\eta}^{2} / \mu^{2}\right)+4 c m_{s}^{2}+4 b m_{s}^{2}
\end{gathered}
$$

6 Note that the $(a)$ superscript on $\Delta^{(a)}$ is not an $S U(3)_{V}$ index! 


$$
\begin{gathered}
\left(P^{*}-P\right)=\Delta-g^{2} \Delta \frac{M_{K}^{2}}{16 \pi^{2} f^{2}} \ln \left(M_{K}^{2} / \mu^{2}\right) \\
-\frac{1}{6} g^{2} \Delta \frac{M_{\eta}^{2}}{16 \pi^{2} f^{2}} \ln \left(M_{\eta}^{2} / \mu^{2}\right)+2 \Delta^{(\sigma)} m_{s} \\
\left\{\left(P_{s}^{*}-P_{s}\right)-\left(P^{*}-P\right)\right\}=-g^{2} \Delta \frac{M_{K}^{2}}{16 \pi^{2} f^{2}} \ln \left(M_{K}^{2} / \mu^{2}\right) \\
-\frac{1}{2} g^{2} \Delta \frac{M_{\eta}^{2}}{16 \pi^{2} f^{2}} \ln \left(M_{\eta}^{2} / \mu^{2}\right)+2 \Delta^{(a)} m_{s} .
\end{gathered}
$$

In the above equations, the chiral logarithms $M_{\eta}^{2} \ln M_{\eta}^{2} / \mu^{2}$ have not been rewritten in terms of $M_{K}$ in order to define the counterterms in a manner consistent with the counterterms which appear in equations derived for the isospin splittings in the next section.

\section{Calculation of Heavy Meson Masses Including $m_{u}$ and $m_{d}$}

In order to compute the isospin splittings amongst the heavy mesons, the calculation of Sect. 3 must be generalized to include nonvanishing $u$ and $d$ quark masses. In addition to isospin splittings due to the $m_{d}-m_{u}$ mass difference, there are isospin splittings due to the difference of electromagnetic charges of the $d$ and $u$ quarks. Electromagnetic effects are expected to contribute significantly to the isospin splittings of the heavy mesons, and must be included. In this section, the formulæ of the previous section are generalized to include nonzero $u$ and $d$ quark masses, and electromagnetic effects.

The appropriate generalizations of eqs. (3.1) and (3.2) to include nonzero masses of the $u$ and $d$ quarks are given by

$$
\begin{aligned}
\frac{1}{4}\left(P_{a}+3 P_{a}^{*}\right) & =m_{H}+\alpha_{a}-\sum_{X=\pi, K, \eta} \beta_{a}^{(X)} \frac{M_{X}^{3}}{16 \pi f^{2}} \\
& +\sum_{X=\pi, K, \eta}\left(\gamma_{a}^{(X)}-\lambda_{a}^{(X)} \alpha_{a}\right) \frac{M_{X}^{2}}{16 \pi^{2} f^{2}} \ln \left(M_{X}^{2} / \mu^{2}\right)+c_{a}
\end{aligned}
$$

and

$$
\left(P_{a}^{*}-P_{a}\right)=\Delta+\sum_{X=\pi, K, \eta}\left(\gamma_{a}^{(X)}-\lambda_{a}^{(X)} \Delta\right) \frac{M_{X}^{2}}{16 \pi^{2} f^{2}} \ln \left(M_{X}^{2} / \mu^{2}\right)+\delta c_{a}
$$

where the sum over pseudo-Goldstone bosons $X$ now includes massive pions, and isospin splittings in the pseudo-Goldstone boson masses are retained. The coefficients appearing 
in eqs. (4.1) and (4.2) are given in the appendix. Isospin splittings are very small, as are mass effects proportional to the $u$ and $d$ quark masses. We will therefore neglect terms which are of order $m_{u}\left(m_{d}-m_{u}\right)$, and $m_{d}\left(m_{d}-m_{u}\right)$ in comparison to effects of order $m_{s}\left(m_{d}-m_{u}\right)$. Finally, note that it is important to include $\pi^{0}-\eta$ mixing in the calculation of loop diagrams, since the mixing angle is proportional to $\left(m_{d}-m_{u}\right)$. Formulæ relevant for the inclusion of this effect are given in the appendix.

There are two isospin splittings, a spin-independent splitting and a spin-dependent splitting. These splittings are determined from eqs. (4.1) and (4.2) and from electromagnetic splittings given in Sect. 2. The spin-independent splitting is given by

$$
\begin{aligned}
& \left\{\frac{1}{4}\left(P_{d}+3 P_{d}^{*}\right)-\frac{1}{4}\left(P_{u}+3 P_{u}^{*}\right)\right\}=2 a\left(m_{d}-m_{u}\right)-g^{2} \frac{\left(M_{K^{0}}^{3}-M_{K^{-}}^{3}\right)}{16 \pi f^{2}} \\
& -g^{2} \sqrt{\frac{4}{3}} \sin \phi \frac{M_{\eta}^{3}}{16 \pi f^{2}}-2 a\left(m_{d}-m_{u}\right)\left(\frac{1}{2}+\frac{3}{2} g^{2}\right) \frac{M_{K}^{2}}{16 \pi^{2} f^{2}} \ln \left(M_{K}^{2} / \mu^{2}\right) \\
& -2 a m_{s}\left(\frac{1}{2}-\frac{3}{2} g^{2}\right)\left[\frac{M_{K^{0}}^{2}}{16 \pi^{2} f^{2}} \ln \left(M_{K^{0}}^{2} / \mu^{2}\right)-\frac{M_{K}^{2}}{16 \pi^{2} f^{2}} \ln \left(M_{K^{-}}^{2} / \mu^{2}\right)\right] \\
& -2 a\left(m_{d}-m_{u}\right)\left(\frac{1}{6}\right) \frac{M_{\eta}^{2}}{16 \pi^{2} f^{2}} \ln \left(M_{\eta}^{2} / \mu^{2}\right)+4 c\left(m_{d}-m_{u}\right) m_{s} \\
& -a_{e m} \alpha_{e m} q_{Q}-\frac{1}{3} b_{e m} \alpha_{e m}
\end{aligned}
$$

and the hyperfine splitting is given by

$$
\begin{aligned}
\left\{\left(P_{d}^{*}-P_{d}\right)-\left(P_{u}^{*}-P_{u}\right)\right\} & =-g^{2} \Delta\left[\frac{M_{K^{0}}^{2}}{16 \pi^{2} f^{2}} \ln \left(M_{K^{0}}^{2} / \mu^{2}\right)-\frac{M_{K^{-}}^{2}}{16 \pi^{2} f^{2}} \ln \left(M_{K^{-}}^{2} / \mu^{2}\right)\right] \\
& -g^{2} \Delta \sqrt{\frac{4}{3}} \sin \phi\left[\frac{M_{\eta}^{2}}{16 \pi^{2} f^{2}} \ln \left(M_{\eta}^{2} / \mu^{2}\right)\right] \\
& +2 \Delta^{(a)}\left(m_{d}-m_{u}\right)-\Delta_{e m}^{(a)} \alpha_{e m} q_{Q}-\frac{1}{3} \Delta_{e m}^{(b)} \alpha_{e m},
\end{aligned}
$$

where $\phi$ is the $\pi^{0}-\eta$ mixing angle as defined in the appendix. In eqs. (4.3) and (4.4), only terms of first order in $\left(m_{d}-m_{u}\right)$ have been retained, and terms proportional to $m_{u, d}\left(m_{d}-m_{u}\right)$ have been neglected compared to terms proportional to $m_{s}\left(m_{d}-m_{u}\right)$. There is a consistency check between the equations eq. (3.13) and eqs. (4.4). The $\mu$ dependence of the $\Delta^{(a)}$ counterterm is the same in both equations. 


\section{Comparison with Experiment}

The experimentally measured mass splittings of mesons containing a single heavy quark are

$$
\begin{aligned}
& \left(D_{s}^{+}-D^{+}\right)=99.5 \pm 0.6 \mathrm{MeV} \\
& \left(D^{+}-D^{0}\right)=4.80 \pm 0.10 \pm 0.06 \mathrm{MeV} \\
& \left(D^{*+}-D^{* 0}\right)=3.32 \pm 0.08 \pm 0.05 \mathrm{MeV} \\
& \left(D^{* 0}-D^{0}\right)=142.12 \pm 0.05 \pm 0.05 \mathrm{MeV} \\
& \left(D^{*+}-D^{+}\right)=140.64 \pm 0.08 \pm 0.06 \mathrm{MeV} \\
& \left(D_{s}^{*+}-D_{s}^{+}\right)=141.5 \pm 1.9 \mathrm{MeV} \\
& \left\{\left(D^{* 0}-D^{0}\right)-\left(D^{*+}-D^{+}\right)\right\}=1.48 \pm 0.09 \pm 0.05 \mathrm{MeV}
\end{aligned}
$$

for the $D$ mesons, and

$$
\begin{aligned}
& \left(B_{s}-B\right)=82.5 \pm 2.5 \mathrm{MeV} \text { or } 121 \pm 9 \mathrm{MeV} \\
& \left(B^{0}-B^{+}\right)=0.01 \pm 0.08 \mathrm{MeV} \text { 23] } \\
& \left(B^{*}-B\right)=46.2 \pm 0.3 \pm 0.8 \mathrm{MeV} \text { 24 or } 45.4 \pm 1.0 \mathrm{MeV} \text { 23 } \\
& \left(B_{s}^{*}-B_{s}\right)=47.0 \pm 2.6 \mathrm{MeV} \text { 23 }
\end{aligned}
$$

for the $B$ mesons. These values can now be compared with the theoretical formulæ eqs. (3.10)-(3.13), (4.3), and (4.4). In some instances, only qualitative statements about the mass splittings can be made because of unknown counterterm parameters. We first discuss the spin-independent splittings, and then the hyperfine splittings.

\section{SPIN-INDEPENDENT SPLITTINGS}

We begin by comparing the experimental data with the theoretical formula for the $S U(3)_{V}$-violating mass splitting $\left\{\frac{1}{4}\left(P_{s}+3 P_{s}^{*}\right)-\frac{1}{4}\left(P_{d}+3 P_{d}^{*}\right)\right\}$ given in eq. (3.11). Only the pseudoscalar splittings $\left(D_{s}^{+}-D^{+}\right)$and $\left(B_{s}-B\right)$ are measured. These pseudoscalar splittings are expected to be equal to the splitting $\left\{\frac{1}{4}\left(P_{s}+3 P_{s}^{*}\right)-\frac{1}{4}\left(P_{d}+3 P_{d}^{*}\right)\right\}$ to an accuracy of a few $\mathrm{MeV}$, since $S U(3)_{V}$ violation in the hyperfine splittings is only of this magnitude. The theoretical formula given in eq. (3.11) implies that the $s-d$ splitting has a contribution which begins at zeroth order in the $1 / m_{Q}$ expansion, i.e. the power series expansion in $1 / m_{Q}$ of the coefficient function $a$ begins at $\mathcal{O}(1)$. If $1 / m_{Q}$ effects are small relative to effects of order unity, then the $s-d$ splitting will be largely independent of the 
mass of the heavy quark. Experimentally, the $\left(D_{s}^{+}-D^{+}\right)$and $\left(B_{s}-B\right)$ are comparable with each of magnitude about $100 \mathrm{MeV}$. The amount of disagreement of the experimental values for the $D$ and $B$ systems is a measure of $1 / m_{Q}$ contributions. Experimentally, the $S U(3)_{V}$ splittings of the $D$ and $B$ mesons differ by roughly $10 \mathrm{MeV}$ (or smaller), which is about a $10 \%$ deviation. The theoretical formula for the $S U(3)_{V}$ splitting has a significant contribution from the $M_{K}^{3}$ term which is independent of the splitting parameter $a$. The $M_{K}^{3}$ term gives a negative contribution to the splitting which can be as big as $-247 \mathrm{MeV}$ for $g^{2} \lesssim 0.5$. The chiral logarithmic correction acts in the opposite direction; for $\mu=1 \mathrm{GeV}$ and $g^{2} \lesssim 0.5$, the parameter $2 a m_{s}$ gets a correction $\lesssim 0.9$ times its tree level value. Thus, the one-loop value of $2 a m_{s}$ can be significantly greater than the value determined at treelevel of approximately $100 \mathrm{MeV}$. In addition, there are two counterterm parameters $b$ and $c$ which cancel the $\mu$ dependence of the chiral logarithm.

The $s-d$ splitting can be compared with the $d-u$ isospin splitting eq. (4.3) which depends on the same parameter $a$. The central value of the isospin splitting $\left\{\frac{1}{4}\left(P_{d}+3 P_{d}^{*}\right)-\frac{1}{4}\left(P_{u}+3 P_{u}^{*}\right)\right\}$ is $3.69 \mathrm{MeV}$ for the $D$ mesons. Only the pseudoscalar isospin splitting is measured for the $B$ mesons; the experimental value is consistent with zero and bounded to be less than a fraction of an MeV. Naively, one expects the isospin splitting to be equal to the $s-d$ splitting times a suppression factor of $\left(m_{d}-m_{u}\right) / m_{s} \approx 1 / 44$. For a $s-d$ splitting of $100 \mathrm{MeV}$, this scaling predicts a $d-u$ splitting of $2.3 \mathrm{MeV}$, which is about the right magnitude. Because the isospin splitting is so small, heavy quark flavor violating and electromagnetic effects which are irrelevant for the $s-d$ splitting can be significant for the isospin splitting. The isospin splittings of the $D$ and $B$ mesons are expected to differ by approximately $2-3 \mathrm{MeV}$ due to electromagnetic effects, eq. (2.16). In addition, the two counterterms $b$ and $c$ relevant for the $s-d$ and isospin splittings have different scaling dependence on the light quark masses. Thus, a more accurate prediction of the isospin splitting from the $s-d$ splitting can not be expected. Evaluation of the $M^{3}$ contribution contributing to the isospin splitting in eq. (4.3) leads to a negative contribution which can be as large as $5.6 \mathrm{MeV}$ for $g^{2} \lesssim 0.5$. The chiral logarithmic corrections to $2 a\left(m_{d}-m_{u}\right)$ are about 0.3 times the tree level value. All these terms are comparable in size to the measured isospin splitting. In summary, the $s-d$ and $d-u$ spin independent splittings are consistent with experiment, but definite predictions cannot be made because of the unknown counterterms $b$ and $c$.

HYPERFINE SPLITTINGS 
The $S U(3)_{V}$ invariant hyperfine splittings of the $D$ and $B$ meson are effects which start at order $1 / m_{Q}$ in the heavy quark mass expansion. The hyperfine splitting for the $D$ mesons is measured to be about $140 \mathrm{MeV}$, whereas the hyperfine splitting is about $46 \mathrm{MeV}$ for the $B$ mesons. The ratio of these two splittings is consistent with what one would expect from naive $1 / m_{Q}$ scaling. The theoretical formulæ for the hyperfine splitting $P^{*}-P$ in eq. (3.13) predicts a chiral logarithmic correction of the tree-level parameter $\Delta$ of $\lesssim 0.15 \Delta$ for $g^{2} \lesssim 0.5$.

The theoretical formulæ eqs. (3.10)-3.13), (4.3) and (4.4) for $S U(3)_{V}$-breaking hyperfine splittings depend on only two parameters, $\Delta$ and a single counterterm proportional to $\Delta^{(a)}$. The leading contribution to the $S U(3)_{V}$ violation in the hyperfine splittings is a chiral logarithmic correction of $\Delta$. For the $s-d$ hyperfine splitting, this correction is $\lesssim 0.2 \Delta \approx 28 \mathrm{MeV}$ for $g^{2} \lesssim 0.5$. The total $s-d$ hyperfine splitting is of order $1 \mathrm{MeV}$, so the counterterm $2 \Delta^{(a)} m_{s}$ must be chosen to be $-27 \mathrm{MeV}$ for a nearly complete cancellation of the chiral logarithm. For the isospin violating $d-u$ hyperfine splitting, the chiral logarithmic correction is $\lesssim 2 \times 10^{-3} \Delta \approx 0.28 \mathrm{MeV}$, and accounts for only a fraction of the hyperfine splitting. Thus most of the isospin hyperfine splitting comes from the counterterm and electromagnetic corrections. The $\Delta^{(a)}$ counterterm contribution to the isospin hyperfine splitting is $\left(m_{d}-m_{u}\right) / m_{s}$ times $-27 \mathrm{MeV}$, or $-0.6 \mathrm{MeV}$. Together, the chiral logarithm and the counterterm account for only approximately $-0.3 \mathrm{MeV}$ of the isospin hyperfine splitting. The measured value of the isospin hyperfine splitting $\left\{\left(\left(D^{*+}-D^{+}\right)-D^{* 0}-D^{0}\right)\right\}=-1.48 \pm 0.09 \pm 0.05$ therefore determines the electromagnetic contribution to the isospin hyperfine splitting to be about $-1.2 \mathrm{MeV}$, which is of the order of magnitude expected for electromagnetic hyperfine splittings. The isospin hyperfine splitting for the $B$ mesons has not been measured. The $1 / m_{Q}$ scaling of $\Delta$ and $\Delta^{(a)}$ implies that the contribution to the $B$ meson hyperfine splittings from the $d-u$ mass difference is of order $-0.3\left(m_{D} / m_{B}\right)=-0.1 \mathrm{MeV}$. The electromagnetic contribution, however, does not scale like $m_{D} / m_{B}$ because of flavor symmetry violation in $\Delta_{e m}^{(a)}$ from the heavy quark charge.

\section{Conclusions}

The experimental data for $D$ and $B$ meson $S U(3)_{V}$ and hyperfine splittings is consistent with the theoretical calculation to one-loop order in chiral perturbation theory. The $1 / m_{Q}$ corrections to the leading predictions for $S U(3)_{V}$ splittings are found to be a 
small correction experimentally. Hyperfine splittings, whose leading dependence on $1 / m_{Q}$ begins at linear order in the $1 / m_{Q}$ expansion, are found to satisfy naive $1 / m_{Q}$ scaling experimentally. Thus, leading order scaling in the the heavy quark mass as implied by the heavy quark mass expansion is evident in the experimental data. Naive $1 / m_{Q}$ scaling implies that

$$
\frac{1}{4}\left\{\left(D_{s}+3 D_{s}^{*}\right)-\left(D+3 D^{*}\right)\right\}=\frac{1}{4}\left\{\left(B_{s}+3 B_{s}^{*}\right)-\left(B+3 B^{*}\right)\right\}
$$

up to corrections of order $1 / m_{Q}$ and that

$$
\frac{\left(B^{*}-B\right)}{\left(D^{*}-D\right)}=\frac{\left\{\left(B_{s}^{*}-B_{s}\right)-\left(B^{*}-B\right)\right\}}{\left\{\left(D_{s}^{*}-D_{s}\right)-\left(D^{*}-D\right)\right\}} .
$$

The two ratios above equal $m_{c} / m_{b}$ to leading order in $m_{Q}$. Generalizations of eqs. (6.1) and (6.2) to the isospin splittings are violated by electromagnetic effects which are significant. Finally, there are a large number of counterterms in the heavy meson lagrangian at one loop, so very few definite predictions which are independent of the values of the counterterms can be obtained. The experimental data can be used to determine the one-loop counterterms once the value of $g^{2}$ is measured.

The most important conclusion of this work, however, is that the $s-d$ and isospin splittings of $D$ and $B$ mesons are consistent with the theory. No breakdown of chiral perturbation theory or the heavy quark mass expansion is in evidence from the theoretical analysis of the $D$ and $B$ meson masses. 


\section{Acknowledgements}

I would like to thank M. Luke, A.V. Manohar, A. Pich, J.L. Rosner, M.J. Savage and M.B. Wise for discussions. I would like to thank M.B. Wise for informing me that he and L. Randall are also studying the non-analytic corrections to the heavy meson masses.

\section{Appendix A. Coefficients for Chiral Expansion in Light Quark Masses}

$$
\begin{aligned}
\frac{1}{4}\left(P_{a}+3 P_{a}^{*}\right) & =m_{H}+\alpha_{a}-\sum_{X=\pi, K, \eta} \beta_{a}^{(X)} \frac{M_{X}^{3}}{16 \pi f^{2}} \\
& +\sum_{X=\pi, K, \eta}\left(\gamma_{a}^{(X)}-\lambda_{a}^{(X)} \alpha_{a}\right) \frac{M_{X}^{2}}{16 \pi^{2} f^{2}} \ln \left(M_{X}^{2} / \mu^{2}\right)+c_{a}
\end{aligned}
$$

Tree-level coefficients:

$$
\alpha_{a}=2 \sigma\left(m_{u}+m_{d}+m_{s}\right)+2 a\left(m_{u} \delta_{1 a}+m_{d} \delta_{2 a}+m_{s} \delta_{3 a}\right)
$$

$m_{q}^{3 / 2}$ coefficients (including isospin violation for $a=1,2$ ):

$$
\begin{array}{lll}
\beta_{1}^{\left(\pi^{0}\right)}=g^{2}\left[\frac{1}{\sqrt{2}} c_{\phi}+\frac{1}{\sqrt{6}} s_{\phi}\right]^{2} & \beta_{2}^{\left(\pi^{0}\right)}=g^{2}\left[-\frac{1}{\sqrt{2}} c_{\phi}+\frac{1}{\sqrt{6}} s_{\phi}\right]^{2} & \\
\beta_{1}^{\left(\pi^{-}\right)}=g^{2} & \beta_{2}^{\left(\pi^{+}\right)}=g^{2} & \beta_{3}^{(\pi)}=0 \\
\beta_{1}^{\left(K^{-}\right)}=g^{2} & \beta_{2}^{\left(K^{0}\right)}=g^{2} & \beta_{3}^{(K)}=2 g^{2} \\
\beta_{1}^{(\eta)}=g^{2}\left[\frac{1}{\sqrt{6}} c_{\phi}-\frac{1}{\sqrt{2}} s_{\phi}\right]^{2} & \beta_{2}^{(\eta)}=g^{2}\left[\frac{1}{\sqrt{6}} c_{\phi}+\frac{1}{\sqrt{2}} s_{\phi}\right]^{2} & \beta_{3}^{(\eta)}=\frac{2}{3} g^{2}
\end{array}
$$

Here $s_{\phi}$ and $c_{\phi}$ are the sine and cosine of the $\pi^{0}-\eta$ mixing angle, where the $\pi^{0}$ and $\eta$ fields in eq. (2.6) are related to the mass eigenstate fields $\pi_{\text {phys }}^{0}$ and $\eta_{\text {phys }}$ by

$$
\begin{aligned}
& \pi_{\text {phys }}^{0}=\cos \phi \pi^{0}+\sin \phi \eta, \\
& \eta_{\text {phys }}=-\sin \phi \pi^{0}+\cos \phi \eta
\end{aligned}
$$

The $\pi^{0}-\eta$ mixing angle is determined to first order in isospin breaking to be

$$
\sin \phi \approx \phi=\frac{\sqrt{3}\left(m_{d}-m_{u}\right)}{4 m_{s}}
$$

Wavefunction renormalization coefficients:

$$
\lambda_{a}^{(X)}=\frac{3}{2} \beta_{a}^{(X)}
$$


One-loop coefficients:

$$
\begin{aligned}
\left(\gamma_{a}^{(\pi)}-\lambda_{a}^{(\pi)} \alpha_{a}\right)= & -a\left[\left(2 m_{u}+m_{d}\right) \delta_{1 a}+\left(m_{u}+2 m_{d}\right) \delta_{2 a}\right] \\
+ & 3 g^{2} a\left[\left(-m_{u}+m_{d}\right) \delta_{1 a}+\left(m_{u}-m_{d}\right) \delta_{2 a}\right] \\
- & 3 \sigma\left(m_{u}+m_{d}\right) \\
\left(\gamma_{a}^{\left(K^{+}\right)}-\lambda_{a}^{\left(K^{+}\right)} \alpha_{a}\right) & =-a\left[\left(m_{u}+m_{s}\right) \delta_{1 a}+\left(m_{u}+m_{s}\right) \delta_{3 a}\right] \\
& +3 g^{2} a\left[\left(m_{s}-m_{u}\right) \delta_{1 a}+\left(m_{u}-m_{s}\right) \delta_{3 a}\right] \\
& -2 \sigma\left(m_{u}+m_{s}\right) \\
\left(\gamma_{a}^{\left(K^{0}\right)}-\lambda_{a}^{\left(K^{0}\right)} \alpha_{a}\right) & =-a\left[\left(m_{d}+m_{s}\right) \delta_{2 a}+\left(m_{d}+m_{s}\right) \delta_{3 a}\right] \\
& +3 g^{2} a\left[\left(m_{s}-m_{d}\right) \delta_{2 a}+\left(m_{d}-m_{s}\right) \delta_{3 a}\right] \\
& -2 \sigma\left(m_{d}+m_{s}\right) \\
& -\frac{1}{3} \sigma\left(m_{u}+m_{d}+4 m_{s}\right) \\
\left(\gamma_{a}^{(\eta)}-\lambda_{a}^{(\eta)} \alpha_{a}\right) & -a\left[\frac{1}{3} m_{u} \delta_{1 a}+\frac{1}{3} m_{d} \delta_{2 a}+\frac{4}{3} m_{s} \delta_{3 a}\right] \\
&
\end{aligned}
$$

Counterterm coefficients:

$$
\begin{aligned}
& c_{a}= 4 d\left(m_{u}^{2}+m_{d}^{2}+m_{s}^{2}\right)+4 c\left(m_{u}+m_{d}+m_{s}\right)\left(m_{u} \delta_{1 a}+m_{d} \delta_{2 a}+m_{s} \delta_{3 a}\right) \\
&+4 b\left(m_{u}^{2} \delta_{1 a}+m_{d}^{2} \delta_{2 a}+m_{s}^{2} \delta_{3 a}\right) \\
&\left(P_{a}^{*}-P_{a}\right)=\Delta+\sum_{X=\pi, K, \eta}\left(\gamma_{a}^{(X)}-\lambda_{a}^{(X)} \Delta\right) \frac{M_{X}^{2}}{16 \pi^{2} f^{2}} \ln \left(M_{X}^{2} / \mu^{2}\right)+\delta c_{a}
\end{aligned}
$$

One-loop coefficients:

$$
\left(\gamma_{a}^{(X)}-\lambda_{a}^{(X)} \Delta\right)=-\Delta\left(\frac{2}{3} \lambda_{a}^{(X)}\right)=-\Delta \beta_{a}^{(X)}
$$

using eq. (A.4).

Counterterm coefficients:

$$
\delta c_{a}=2 \Delta^{(\sigma)}\left(m_{u}+m_{d}+m_{s}\right)+2 \Delta^{(a)}\left(m_{u} \delta_{1 a}+m_{d} \delta_{2 a}+m_{s} \delta_{3 a}\right)
$$




\section{References}

[1] N. Isgur and M.B. Wise, Phys. Lett. 232B (1989) 113;

Phys. Lett. 237B (1990) 527

[2] A.F. Falk, B. Grinstein and M.E. Luke, Nucl. Phys. B357 (1991) 185

[3] J.L. Goity, CEBAF-TH-92-16 [hep-ph/9206230

[4] J.L. Rosner and M.B. Wise, CALT-68-1807

[5] J.L. Goity, CEBAF-TH-92-26 [hep-ph/9212230]

[6] L. Randall and E. Sather, MIT-CTP\#2166

[7] H. Georgi, Heavy Quark Effective Field Theory, in Proceedings of the Theoretical Advanced Study Institute 1991, ed. R.K. Ellis, C.T. Hill and J.D. Lykken, World Scientific (1992)

[8] H. Georgi, Phys. Lett. 240B (1990) 447

[9] M.B. Wise, Phys. Rev. D45 (1992) 2188

[10] G. Burdman and J.F. Donoghue, Phys. Lett. 280B (1992) 287

[11] T.M. Yan, H.Y. Cheng, C.Y. Cheung, G.L. Lin, Y.C. Lin and H.L. Yu, Phys. Rev. D46 (1992) 1148

[12] E. Jenkins and A.V. Manohar, Phys. Lett. 255B (1991) 558

[13] E. Jenkins and A.V. Manohar, Baryon Chiral Perturbation Theory, in Proceedings of the Workshop on "Effective Field Theories of the Standard Model," ed. U. Meissner, World Scientific (1992)

[14] A.V. Manohar and H. Georgi, Nucl. Phys. B234 (1984) 189

[15] The ACCMOR Collaboration (S. Barlag et al.), Phys. Lett. 278B (1992) 480

[16] The CLEO Collaboration (F. Butler et al.), Phys. Rev. Lett. 69 (1992) 2041

[17] J.F. Amundson, C.G. Boyd, E. Jenkins, M. Luke, A.V. Manohar, J.L. Rosner, M.J. Savage and M.B. Wise, hep-ph/9209241 ;

P. Cho and H. Georgi, HUTP-92/A043 hep-ph/9209239;

H.Y. Cheng, C.Y. Cheung, G.L. Lin, Y.C. Lin, T.M. Yan and H.L. Yu, CLNS 92/1158

[18] P. Cho, HUTP-92/A039 hep-ph/9208244

[19] J. Gasser and H. Leutwyler, Phys. Rep. 87 (1982) 77

[20] E. Jenkins, Nucl. Phys. B368 (1992) 190

[21] Particle Data Group, Phys. Rev. D45 (1992)

[22] The Cleo Collaboration (D. Bortoletto et al.) Phys. Rev. Lett. 69 (1992) 2046

[23] The CUSB-II Collaboration (J. Lee-Franzini et al.), Phys. Rev. Lett. 65 (1990) 2947

[24] The Cleo II Collaboration (D.S. Akerib et al.), Phys. Rev. Lett. 67 (1991) 1692 


\section{Figure Captions}

Fig. 1. Tree-level heavy meson mass splittings arising from $\mathcal{L}_{v}^{M}$ and $\mathcal{L}_{v}^{\phi}$. Solid lines denote the $H_{v}$ field. The solid triangle (a) denotes the $S U(3)_{V}$-violating vertex in $\mathcal{L}_{v}^{M}$ proportional to $a$. The vertex results in a $\left(P_{s}^{(*)}-P^{(*)}\right)$ mass splitting which is spin independent. The solid diamond (b) represents the vertex proportional to $\sigma$ in $\mathcal{L}_{v}^{M}$ which yields a singlet contribution to the pseudoscalar and vector meson masses. The solid square vertex (c) represents the vertex in $\mathcal{L}_{v}^{\phi}$. The vertex leads to a $S U(3)_{V}$ flavor-independent $\left(P_{a}^{*}-P_{a}\right)$ spin splitting $\Delta$ which violates heavy quark spin flavor symmetry.

Fig. 2. One-loop diagrams which contribute to the heavy meson masses. Solid dots denote the axial pion-heavy meson vertex proportional to $g$ contained in $\mathcal{L}_{v}^{0}$. Diagram (a) is responsible for the non-analytic $m_{s}^{3 / 2}$ mass contribution and for wavefunction renormalization coefficients $\lambda_{a}$. Graphs (b) and (c) yield the coefficients $\gamma_{a}$ which occur in the $m_{s}^{2} \ln m_{s}$ non-analytic contributions to the heavy meson masses. Diagrams (b) are proportional to $a$, and diagrams (c) are proportional to $\sigma$. Graph (d) yields the coefficients $\gamma_{a}$ which parameterize the $\Delta m_{s} \ln m_{s}$ contribution to the heavy meson masses. This non-analytic contribution violates both $S U(3)_{V}$ flavor symmetry and heavy quark spin flavor symmetry, and yields the leading contribution to the mass difference $\left\{\left(P_{s}^{*}-P_{s}\right)-\left(P^{*}-P\right)\right\}$. 
